Bull. Austral. Math. Soc.

VOL. 69 (2004) [519-528]

\title{
AN INEQUALITY WITH APPLICATION \\ TO A DIFFERENCE EQUATION
}

\section{Jong-Yi Chen ANd Yunshyong Chow}

In this paper we shall prove that for any $0<d \leqslant 2$,

$$
\sum_{j=1}^{n}\left(\sum_{s=j}^{n} s^{(2 / d)-1}\right)^{-(d / 2)} \leqslant \sum_{j=1}^{n+1}\left(\sum_{s=j}^{n+1} s^{(2 / d)-1}\right)^{-d / 2} \text { holds for } n \geqslant 1 .
$$

As an application, we shall then show that the following recursively defined sequence

$$
\tau_{1}=1 \text { and } \sum_{j=1}^{n}\left(\sum_{s=j}^{n} \tau_{s}\right)^{-d / 2}=1 \text { for } n \geqslant 2
$$

satisfies

$$
\lim _{n \rightarrow \infty} \tau_{n} / n^{(2 / d)-1}=\left(\frac{d}{2}\right)^{-1+(2 / d)}\left(\pi / \sin \frac{d \pi}{2}\right)^{2 / d} \text { for } 0<d<2
$$

The difference equation above originates from a heat conduction problem studied by Myshkis (J. Difference Equ. Appl. 3(1997), 89-91).

\section{INTRODUCTION AND RESULTS}

The main purpose of this paper is to show the following inequality.

THEOREM 1.1. Let $0<d \leqslant 2$ be fixed. Then

$$
\sum_{j=1}^{n}\left(\sum_{s=j}^{n} s^{(2 / d)-1}\right)^{-d / 2} \leqslant \sum_{j=1}^{n+1}\left(\sum_{s=j}^{n+1} s^{(2 / d)-1}\right)^{-d / 2} \quad \text { holds for } n \geqslant 1
$$

Equation (1.1) is trivial for $d=2$. It does not seem obvious for the remaining cases. We shall first prove a series of elementary inequalities some of which are interesting by themselves.

Received 5th January, 2004

The authors were partially supported by the National Science Council, Repubic of China. Thanks to an anonymous referee for pointing out the usage of Beta function and Gamma function. This improves greatly the statement of Theorem 1.3.

Copyright Clearance Centre, Inc. Serial-fee code: 0004-9727/04 \$A2.00+0.00. 
LEMmA 1.2. Let $\sigma \geqslant 1$ be fixed and $A_{n}=\sum_{s=1}^{n} s^{\sigma-1}$. Then for $n \in \mathrm{N}$,

(i) $\max \left(\left(\frac{n+1}{n+2}\right)^{\sigma-1}(n+1)-\left(\frac{n}{n+1}\right)^{\sigma-1} n,\left(\frac{n+2}{n+1}\right)^{\sigma}(n+1)-\left(\frac{n+1}{n}\right)^{\sigma} n\right)$ $\leqslant 1$,

(ii) $\frac{n^{\sigma}(n+1)^{\sigma-1}}{(n+1)^{\sigma}-n^{\sigma}} \leqslant A_{n} \leqslant \frac{(n+1)^{2 \sigma-1}}{(n+2)^{\sigma}-(n+1)^{\sigma}}-\frac{1}{2^{\sigma}-1}$,

(iii) $\frac{n^{\sigma-1}}{A_{n}} \geqslant \frac{(n+1)^{\sigma-1}}{A_{n+1}}, \frac{n^{\sigma}}{A_{n}} \leqslant \frac{(n+1)^{\sigma}}{A_{n+1}}$ and $\frac{(n+1)^{\sigma}}{A_{n}} \geqslant \frac{(n+2)^{\sigma}}{A_{n+1}}$,

(iv) $\frac{A_{n+1}}{A_{n}} \geqslant \frac{A_{n+2}}{A_{n+1}}$

(v) both sequences $\left\{\frac{A_{n+1}}{A_{n}^{1-(1 / \sigma)}}-A_{n}^{1 / \sigma}\right\}$ and $\left\{\frac{A_{n+1}}{A_{n}}\left(A_{n+1}^{1 / \sigma}-A_{n}^{1 / \sigma}\right)\right\}$ are decreasing in $n$,

(vi) the positive sequence $\left\{A_{n+1}^{1 / \sigma}\left(\frac{A_{n+1}}{A_{n}}\right)^{k}-A_{n}^{1 / \sigma}\right\}$ is decreasing in $n$ for each integer $k \geqslant 1$,

(vii) the sequence $\left\{\left(\sum_{j=1}^{n} A_{j-1}^{k}\right) / A_{n}^{k+(1 / \sigma)}\right\}$ is increasing in $n$ for each integer $k \geqslant 0$.

Assuming Lemma 1.2 tentatively, we can prove Theorem 1.1 easily. Obviously, (1.1) is equivalent to showing $D_{n}$, the sum on the left of (1.1), is increasing in $n$. Let $A_{0}=0$ and $A_{n}=\sum_{s=1}^{n} s^{(2 / d)-1}$ for $n \geqslant 1$. Using $\sum_{s=j}^{n} s^{(2 / d)-1}=A_{n}-A_{j-1}$ and the Binomial Theorem,

$$
\begin{aligned}
D_{n} & =\sum_{j=1}^{n}\left(A_{n}-A_{j-1}\right)^{-d / 2}=A_{n}^{-d / 2} \sum_{j=1}^{n}\left(1-\frac{A_{j-1}}{A_{n}}\right)^{-d / 2} \\
& =A_{n}^{-d / 2} \sum_{j=1}^{n}\left(\sum_{k=0}^{\infty}(-1)^{k}\left(\begin{array}{c}
-\frac{d}{2} \\
k
\end{array}\right)\left(\frac{A_{j-1}}{A_{n}}\right)^{k}\right)=\sum_{k=0}^{\infty}(-1)^{k}\left(\begin{array}{c}
-\frac{d}{2} \\
k
\end{array}\right) \sum_{j=1}^{n} A_{j-1}^{k} / A_{n}^{k+(d / 2)} .
\end{aligned}
$$

Since $(-1)^{k}\left(\begin{array}{c}-d / 2 \\ k\end{array}\right)>0$ for $k \geqslant 0,(1.1)$ follows immediately from Lemma 1.2 (vii) with $\sigma$ there replaced by $2 / d$. This verifies Theorem 1.1 .

As an application of Theorem 1.1, we consider the following difference equation:

$$
\tau_{1}=1 \text { and } \sum_{j=1}^{n}\left(\sum_{s=j}^{n} \tau_{s}\right)^{-d / 2}=1 \text { for } n \geqslant 2 .
$$

The asymptotic behaviour of the sequence $\left\{\tau_{n}\right\}$ for $0<d<2$ is answered as follows. 
THEOREM 1.3. $\lim _{n \rightarrow \infty} \tau_{n} / n^{(2 / d)-1}=(d / 2)^{-1+(2 / d)}(\pi / \sin (d \pi / 2))^{2 / d}$ for $0<d$
$<2$.

The difference equation (1.2) originates from the following heat conduction problem studied by Myshkis [4]: Let $u(\mathrm{x}, t)$ be the temperature at position $\mathrm{x}=\left(x_{1}, x_{2}, \ldots, x_{d}\right)$ and time $t$ of a homogeneous medium filling up all of $\mathbf{R}^{d}$. Suppose $u \equiv 0$ at $t=0$ and a heat impulse of size $b$ is applied at $\mathbf{x}=0$ and the temperature there is increased to above a fixed temperature $u_{0}>0$. When the temperature at the origin decays to the value $u_{0}$ at time $t_{1}$, a heat impulse of the same size is applied again at the origin. This process of applying heat impulses so the temperature at the origin never goes below $u_{0}$ is repeated indefinitely.

Denote by $t_{0}=0, t_{1}, t_{2}, \ldots$ the sequence of consecutive times that a heat impulse of size $b$ is applied at $\mathbf{x}=0$. By solving the heat equation

$$
\left\{\begin{array}{l}
\frac{\partial u}{\partial t}=a \sum_{i=1}^{d} \frac{\partial^{2} u}{\partial x_{i}^{2}} \\
u\left(\mathbf{x}, t_{n}^{+}\right)=u\left(\mathbf{x}, t_{n}\right)+b \delta(\mathbf{x})
\end{array}\right.
$$

where $a$ is the heat conduction coefficient of the medium, it is not difficult to obtain that for $n \geqslant 0$ and $t_{n}<t \leqslant t_{n+1}$,

$$
u(\mathrm{x}, t)=b \sum_{j=0}^{n}\left(\frac{1}{4 \pi a\left(t-t_{j}\right)}\right)^{d / 2} \exp \left(-\frac{\sum_{i=1}^{d} x_{i}^{2}}{4 a\left(t-t_{j}\right)}\right)
$$

Define $\tau_{n}=4 \pi a\left(t_{n}-t_{n-1}\right)\left(u_{0} / b\right)^{2 / d}$ for $n \geqslant 1$. Since $u\left(0, t_{n}\right)=u_{0}$, a simple computation leads to (1.2). The sequence $\left\{\tau_{n}\right\}$ is thus recursively defined. Yet it is difficult to solve (1.2) for $\tau_{n}$. Myshkis [4] proposed as an open problem the asymptotic expression for $\tau_{n}$ not only for $d=1$ but also for more general settings. Note that sequence $\left\{\tau_{n}\right\}$ is well defined for any $d>0$. Chen, Chow and Hsieh [2] proved that $\lim _{n} \tau_{n} / n=\pi^{2} / 2$ for $d=1$, a special case of Theorem 1.3, by first establishing the following lemma.

LEMMA 1.4. [2]

(i) The sequence $\left\{\tau_{n}\right\}$ defined in (1.2) is increasing for any fixed $d>0$.

(ii) $\sum_{j=1}^{n}\left(\sum_{s=j}^{n} s\right)^{-1 / 2} \leqslant \sum_{j=1}^{n+1}\left(\sum_{s=j}^{n+1} s\right)^{-1 / 2}$ for $n \geqslant 1$.

The increasing property of the sequence $\left\{\tau_{n}\right\}$ shown in Lemma 1.4 (i) is also crucial in this paper. The inequality (1.1) is a generalisaion of that in Lemma 1.4 (ii). Recently Chang, Chow and Wang [1] proved that

$$
\lim _{n} \tau_{n} / \log n=1 \text { for } d=2
$$


by a clever method which unfortunately seems valid only for $d=2$. Note that (1.3) can also be proved by first establishing the following inequality

$$
\sum_{j=1}^{n}\left(\sum_{s=j}^{n} \log s\right)^{-1} \geqslant \sum_{j=1}^{n+1}\left(\sum_{s=j}^{n+1} \log s\right)^{-1} \text { for } n \geqslant 2 \text {. }
$$

Let $\zeta(s)=\sum_{k=1}^{\infty} k^{-s}$ be the Riemann zeta function. It follows easily from Lemma 1.4 (i) that

$$
\lim _{n} \tau_{n}=\zeta(d / 2)^{2 / d} \text { for } d>2
$$

So in this paper we need only to consider the case $0<d<2$. It is not known whether (1.2) for non-integer $d$ bears any physical meaning. Still, it is interesting mathematically to see how one can obtain analytic results for such highly nonlinear difference equations.

Lemma 1.2 is proved in Section 2. As shown above the inequality (1.1) follows easily as a consequence. It takes the place of the inequality in Lemma 1.4 (ii). Finally Theorem 1.3 is shown in Section 3 by following the same arguments in Chen, Chow and Hsieh [2] for the case $d=1$.

\section{Proof of Lemma 1.2}

PART (i). Denote both terms inside the bracket by $\tau(\sigma, n)$ and $\eta(\sigma, n)$ respectively. Let $f(x)=x \log (x /(x+1))$. A simple calculation shows $f^{\prime}(x)=1 /(x+1)+\log (1$ $-(1 / x+1)) \leqslant 0$ for $x>0$. Hence $f(n+1) \leqslant f(n)$. That is

$$
(n+1) \log \left(\frac{n+1}{n+2}\right) \leqslant n \log \left(\frac{n}{n+1}\right) \leqslant 0 .
$$

Using $((n+1) /(n+2))^{\sigma-1} \geqslant(n /(n+1))^{\sigma-1} \geqslant 0$, we get from (2.1) that

$$
\left(\frac{n+1}{n+2}\right)^{\sigma-1}(n+1) \log \left(\frac{n+1}{n+2}\right) \leqslant\left(\frac{n}{n+1}\right)^{\sigma-1} n \log \left(\frac{n}{n+1}\right)
$$

which means $\frac{\partial \tau}{\partial \sigma}(\sigma, n) \leqslant 0$. Hence $\tau(\sigma, n) \leqslant \tau(1, n)=1$ as desired. Introducing $\tilde{f}(x)=(x+1) \log ((x+1) / x)$, we can show in the same way that $\frac{\partial \eta}{\partial \sigma}(\sigma, n) \leqslant 0$ and thus $\eta(\sigma, n) \leqslant \eta(1, n)=1$. The details is omitted.

Note that $\tau(\sigma, n) \leqslant 1$ is the same as $n+1-\left(n^{\sigma}\right) /\left((n+1)^{\sigma-1}\right) \leqslant n+2$ $-(n+1)^{\sigma} /((n+2))^{\sigma-1}$. Or equivalently,

$$
\frac{(n+1)^{\sigma}-n^{\sigma}}{(n+1)^{\sigma-1}} \leqslant \frac{(n+2)^{\sigma}-(n+1)^{\sigma}}{(n+2)^{\sigma-1}} \text {. }
$$


By expansion, $\eta(\sigma, s) \leqslant 1$ implies $(s+1) s^{\sigma}(s+2)^{\sigma}-s(s+1)^{2 \sigma} \leqslant s^{\sigma}(s+1)^{\sigma}$. Replacing the term $s^{\sigma}(s+1)^{\sigma}$ above by $s^{\sigma}(s+1)^{\sigma+1}-s^{\sigma+1}(s+1)^{\sigma}$, a simple rearrangement shows $s(s+1)^{\sigma}\left((s+1)^{\sigma}-s^{\sigma}\right) \geqslant s^{\sigma}(s+1)\left((s+2)^{\sigma}-(s+1)^{\sigma}\right)$. Multiplying both sides by $s^{-1}(s+1)^{\sigma-1}$, we shall get

$$
\frac{(s+1)^{2 \sigma-1}}{(s+2)^{\sigma}-(s+1)^{\sigma}} \geqslant \frac{s^{\sigma-1}(s+1)^{\sigma}}{(s+1)^{\sigma}-s^{\sigma}}=\frac{s^{2 \sigma-1}}{(s+1)^{\sigma}-s^{\sigma}}+s^{\sigma-1} \text { for } s \geqslant 1 .
$$

PART (ii). Since $A_{1}=1 \geqslant\left(2^{\sigma-1}\right) /\left(2^{\sigma}-1\right)$, the first inequality holds for $n=1$. Suppose it holds for $n$. By induction it suffices to check it for $n+1$. Using $A_{n+1}$ $=A_{n}+(n+1)^{\sigma-1}$ and $(2.2)$,

$$
A_{n+1} \geqslant \frac{n^{\sigma}(n+1)^{\sigma-1}}{(n+1)^{\sigma}-n^{\sigma}}+(n+1)^{\sigma-1}=\frac{(n+1)^{2 \sigma-1}}{(n+1)^{\sigma}-n^{\sigma}} \geqslant \frac{(n+1)^{\sigma}(n+2)^{\sigma-1}}{(n+2)^{\sigma}-(n+1)^{\sigma}}
$$

as desired. Using telescoping cancellation, the second inequality follows from adding up (2.3) for $1 \leqslant s \leqslant n$.

PART (iii). The first inequality is shown as follows:

$$
(n+1)^{\sigma-1} A_{n}=\sum_{s=1}^{n}(s(n+1))^{\sigma-1} \leqslant \sum_{s=1}^{n}(n(s+1))^{\sigma-1} \leqslant n^{\sigma-1} A_{n+1} .
$$

Using $A_{n+1}=A_{n}+(n+1)^{\sigma-1}$, a simple rearrangement shows the other two inequalities are equivalent to

$$
\frac{n^{\sigma}(n+1)^{\sigma-1}}{(n+1)^{\sigma}-n^{\sigma}} \leqslant A_{n} \leqslant \frac{(n+1)^{2 \sigma-1}}{(n+2)^{\sigma}-(n+1)^{\sigma}}
$$

which follows immediately from Part (ii).

PART (iv). Using $A_{n+1}=A_{n}+(n+1)^{\sigma-1}$ and $A_{n+2}=A_{n}+(n+1)^{\sigma-1}+(n+2)^{\sigma-1}$, the inequality is equivalent to

$$
A_{n}\left(A_{n}+(n+1)^{\sigma-1}+(n+2)^{\sigma-1}\right)=A_{n} A_{n+2} \leqslant A_{n+1}^{2}=\left(A_{n}+(n+1)^{\sigma-1}\right)^{2} .
$$

By expansion, that is the same as

$$
A_{n} \leqslant \frac{(n+1)^{2 \sigma-2}}{(n+2)^{\sigma-1}-(n+1)^{\sigma-1}}=\frac{(n+1)^{2 \sigma-1}}{(n+1 / n+2)(n+2)^{\sigma}-(n+1)^{\sigma}},
$$

which follows immediately from the second inequality in Part (ii). 
PART (v). The first assertion follows from Part (iii) and the fact that

$$
\left(\frac{(n+1)^{\sigma}}{A_{n}}\right)^{1-(1 / \sigma)}=\frac{(n+1)^{\sigma-1}}{A_{n}^{1-(1 / \sigma)}}=\frac{A_{n+1}-A_{n}}{A_{n}^{1-(1 / \sigma)}}=\frac{A_{n+1}}{A_{n}^{1-(1 / \sigma)}}-A_{n}^{1 / \sigma} .
$$

By using $A_{n+1}=A_{n}+(n+1)^{\sigma-1}$ and the Binomial Theorem, we have

$$
\begin{aligned}
\frac{A_{n+1}}{A_{n}}\left(A_{n+1}^{1 / \sigma}-A_{n}^{1 / \sigma}\right) & =\frac{A_{n+1}^{1+(1 / \sigma)}}{A_{n}}\left(1-\left(1-\frac{(n+1)^{\sigma-1}}{A_{n+1}}\right)^{1 / \sigma}\right) \\
& =\frac{A_{n+1}^{1+(1 / \sigma)}}{A_{n}} \sum_{k=1}^{\infty}(-1)^{k+1}\left(\begin{array}{c}
\frac{1}{\sigma} \\
k
\end{array}\right)\left(\frac{(n+1)^{\sigma-1}}{A_{n+1}}\right)^{k} \\
& =\left(\frac{A_{n+1}}{(n+1)^{\sigma}}\right)^{1 / \sigma} \cdot \frac{(n+1)^{\sigma}}{A_{n}} \sum_{k=1}^{\infty}(-1)^{k+1}\left(\begin{array}{l}
\frac{1}{\sigma} \\
k
\end{array}\right)\left(\frac{(n+1)^{\sigma-1}}{A_{n+1}}\right)^{k-1} .
\end{aligned}
$$

Because $0<1 / \sigma \leqslant 1,(-1)^{k+1}\left(\begin{array}{c}1 / \sigma \\ k\end{array}\right) \geqslant 0$ for $k \geqslant 1$, the second assertion follows from Part (iii).

PART (vi). Because $A_{n}$ is increasing in $n$, each term of the sequence is obviously positive. For $k=1$ we have

$$
A_{n+1}^{1 / \sigma}\left(\frac{A_{n+1}}{A_{n}}\right)-A_{n}^{1 / \sigma}=\left(\frac{A_{n+1}}{A_{n}}\left(A_{n+1}^{1 / \sigma}-A_{n}^{1 / \sigma}\right)\right)+\left(\frac{A_{n+1}}{A_{n}} A_{n}^{1 / \sigma}-A_{n}^{1 / \sigma}\right),
$$

which is decreasing by Part (v). Suppose it holds for $k=\ell$. For $k=\ell+1$ we have

$$
A_{n+1}^{1 / \sigma}\left(\frac{A_{n+1}}{A_{n}}\right)^{\ell+1}-A_{n}^{1 / \sigma}=\left(\frac{A_{n+1}}{A_{n}}\right) \cdot\left(A_{n+1}^{1 / \sigma}\left(\frac{A_{n+1}}{A_{n}}\right)^{\ell+1}-A_{n}^{1 / \sigma}\right)+\left(\frac{A_{n+1}}{A_{n}^{1-(1 / \sigma)}}-A_{n}^{1 / \sigma}\right) .
$$

Since all three terms on the right hand side above are positive and decreasing in $n$ by Parts (iv), (v) and the induction hypothesis, this verifies the case $k=\ell+1$ and thus completes the proof by induction.

PART (vii). Denote the sequence by $\left\{c_{n}\right\}$. The case $k=0$ follows immediately from taking the $\sigma$ th root of the second inequality in Part (iii). For a fixed $k \geqslant 1$ it suffices to prove by induction on $n$ that

$$
c_{n} \leqslant c_{n+1}
$$

A simple calculation after multiplying both sides by $A_{n+1}^{k+(1 / \sigma)}$ shows $(2.4)$ is equivalent to

$$
\sum_{j=1}^{n} A_{j-1}^{k}\left(\left(\frac{A_{n+1}}{A_{n}}\right)^{k+(1 / \sigma)}-1\right) \leqslant A_{n}^{k}
$$


Since $c_{n} \geqslant 0$ and $c_{1}=0$ as $A_{0}=0$ by convention, (2.4) holds for $n=1$. Suppose (2.4) and thus (2.5) hold for $n$. Then by (2.5) for $n$ and Part (vi),

$$
\begin{aligned}
& \sum_{j=1}^{n+1} A_{j-1}^{k}\left(\left(\frac{A_{n+2}}{A_{n+1}}\right)^{k+(1 / \sigma)}-1\right)=A_{n}^{k}\left(\left(\frac{A_{n+2}}{A_{n+1}}\right)^{k+(1 / \sigma)}-1\right) \\
& \quad+\sum_{j=1}^{n} A_{j-1}^{k}\left(\left(\frac{A_{n+2}}{A_{n+1}}\right)^{k+(1 / \sigma)}-1\right) \\
& \leqslant A_{n}^{k}\left(\left(\frac{A_{n+2}}{A_{n+1}}\right)^{k+(1 / \sigma)}-1\right)+A_{n}^{k}\left(\left(\frac{A_{n+2}}{A_{n+1}}\right)^{k+(1 / \sigma)}-1\right) /\left(\left(\frac{A_{n+1}}{A_{n}}\right)^{k+(1 / \sigma)}-1\right) \\
& =A_{n}^{k}\left(\left(\frac{A_{n+2}}{A_{n+1}}\right)^{k+(1 / \sigma)}-1\right) \cdot\left(\frac{A_{n+1}}{A_{n}}\right)^{k+(1 / \sigma)} /\left(\left(\frac{A_{n+1}}{A_{n}}\right)^{k+(1 / \sigma)}-1\right) \\
& =A_{n+1}^{k} \cdot\left(A_{n+2}^{1 / \sigma}\left(\frac{A_{n+2}}{A_{n+1}}\right)^{k}-A_{n+1}^{1 / \sigma}\right) /\left(A_{n+1}^{1 / \sigma}\left(\frac{A_{n+1}}{A_{n}}\right)^{k}-A_{n}^{1 / \sigma}\right) \leqslant A_{n+1}^{k},
\end{aligned}
$$

which means (2.5) and thus (2.4) hold for $n+1$. This completes the proof by induction.

\section{Proof of ThEOREM 1.3}

Let $r=d / 2, \tilde{T}_{j}^{k}=\sum_{s=j}^{k} \tau_{s}$ and $T_{j}^{k}=\sum_{s=j}^{k} s^{(1 / r)-1}$. With the inequality (1.1) at hand, we can follow the same arguments used in Chen, Chow and Hsieh [2] for the case $d=1$. The proof is divided into four steps:

(a) If $n \in S_{c}$ then $m \in S_{c}$ for all $m \geqslant n$, where

$$
S_{c}=\left\{k \in \mathbf{N}: k \geqslant 1 \text { and } \tilde{T}_{j}^{k} \geqslant c T_{j}^{k} \text { for } j=1,2, \ldots, k\right\} .
$$

(b) $\liminf _{n} \tau_{n} / \dot{n}^{(1 / r)-1}=\sup \left\{c: S_{c} \neq \phi\right\} \geqslant 1$

(c) $\limsup \tau_{n} \tau_{n} n^{(1 / r)-1}=\liminf _{n} \tau_{n} / n^{(1 / r)-1}$. Hence $\lim _{n} \tau_{n} / n^{(1 / r)-1}$ exists in $(0, \infty]$.

(d) $\lim _{n} \tau_{n} / n^{(1 / r)-1}=r^{-1+(1 / r)}(\pi / \sin r \pi)^{1 / r}$.

STEP (a). Using $\tilde{T}_{j}^{n+1}=\tau_{n+1}+\widetilde{T}_{j}^{n}$ and (1.2), we have

$$
\sum_{j=1}^{n}\left\{\left(\widetilde{T}_{j}^{n}\right)^{-r}-\left(\tau_{n+1}+\widetilde{T}_{j}^{n}\right)^{-r}\right\}=1-\sum_{j=1}^{n}\left(\widetilde{T}_{j}^{n+1}\right)^{-r}=\left(\tau_{n+1}\right)^{-r} .
$$

Define $h(x)=x^{-r}-(1+x)^{-r}$ for $x>0$. By multiplying $\tau_{n+1}^{r}$ to both sides of (3.1),

$$
\sum_{j=1}^{n} h\left(\tilde{T}_{j}^{n} / \tau_{n+1}\right)=1 \text { for } n \geqslant 1 .
$$


Repeating the same to the sequence $\left\{T_{j}^{n}\right\}$ and using the inequality (1.1), we get

$$
\sum_{j=1}^{n} h\left(T_{j}^{n} /(n+1)^{(1 / r)-1}\right) \leqslant 1 \text { for } n \geqslant 1 .
$$

It suffices to show $n+1 \in S_{c}$ as we shall have successively $n+2 \in S_{c}, n+3 \in S_{c}, \ldots$, and so on. Since $n \in S_{c}$ by assumption,

$$
\widetilde{T}_{j}^{n} \geqslant c T_{j}^{n} \text { holds for } j=1,2, \ldots, n .
$$

It is easy to see that $h$ is a decreasing function. By (3.2), (3.3) and (3.4),

$$
\sum_{j=1}^{n} h\left(T_{j}^{n} /\left(\tau_{n+1} / c\right)\right) \geqslant \sum_{j=1}^{n} h\left(\widetilde{T}_{j}^{n} / \tau_{n+1}\right)=1 \geqslant \sum_{j=1}^{n} h\left(T_{j}^{n} /(n+1)^{(1 / r)-1}\right) .
$$

Certainly $\sum_{j=1}^{n} h\left(x T_{j}^{n}\right)$ is decreasing in $x$. The inequality above implies $\tau_{n+1} / c$ $\geqslant(n+1)^{(1 / r)-1}$. That is $\tau_{n+1} \geqslant c(n+1)^{(1 / r)-1}$. Using $\widetilde{T}_{j}^{n+1}=\tau_{n+1}+\widetilde{T}_{j}^{n}$ and $T_{j}^{n+1}=T_{j}^{n}+(n+1)^{(1 / \tau)-1}$ we conclude from (3.4) that $n+1 \in S_{c}$. This completes the proof of (a). In particular, $\tau_{m}=\widetilde{T}_{m}^{m} \geqslant c T_{m}^{m}=c m^{(1 / r)-1}$ for all $m \geqslant n$. Hence

$$
\liminf _{n} \tau_{n} / n^{(1 / r)-1} \geqslant \sup \left\{c: S_{c} \neq \phi\right\} \geqslant 1 .
$$

The last inequality is due to the fact that $\tau_{1}=1$, which implies $1 \in S_{c}$ where $c=1$.

STEP (b). Let $\alpha=\liminf _{n} \tau_{n} / n^{(1 / r)-1}$. By (3.5), it remains to show

$$
\alpha \leqslant \sup \left\{c: S_{c} \neq \phi\right\} .
$$

For any $\varepsilon>0$, there exists $n_{0} \geqslant 1$ such that

$$
\tau_{m} \geqslant(\alpha-\varepsilon) m^{(1 / r)-1} \text { for } m \geqslant n_{0} .
$$

It suffices to show $S_{\alpha-2 \varepsilon} \neq \phi$. By (3.7), $\widetilde{T}_{j}^{n} \geqslant(\alpha-2 \varepsilon) T_{j}^{n}$ holds for $j \geqslant n_{0}$. Moreover,

$$
\widetilde{T}_{n_{0}}^{n}-(\alpha-2 \varepsilon) T_{n_{0}}^{n} \geqslant \varepsilon T_{n_{0}}^{n} \text { holds for } n \geqslant n_{0} .
$$

For $1 \leqslant j<n_{0}$,

$$
\widetilde{T}_{j}^{n_{0}-1}-(\alpha-2 \varepsilon) T_{j}^{n_{0}-1} \geqslant-(\alpha-2 \varepsilon) T_{j}^{n_{0}-1} \geqslant-(\alpha-2 \varepsilon) T_{1}^{n_{0}-1} .
$$

We obtain, by adding up these two formulas above, that $\widetilde{T}_{j}^{n} \geqslant(\alpha-2 \varepsilon) T_{j}^{n}$ holds for $j<n_{0}$ as well if $n$ is taken so large that $\varepsilon T_{n_{0}}^{n} \geqslant(\alpha-2 \varepsilon) T_{1}^{n_{0}-1}$. Note that $\lim _{n} T_{n_{0}}^{n}$ $=\infty$. Hence $n \in S_{\alpha-2 \varepsilon}$ by definition. In particular, $S_{\alpha-2 \varepsilon} \neq \phi$. This verifies (3.6) and thus (b). 
$\operatorname{STEP}$ (c). Suppose the contrary that $\beta \equiv \lim \sup _{n} \tau_{n} / n^{(1 / r)-1} \geq \liminf _{n} \tau_{n} / n^{(1 / r)-1}$ $\equiv \alpha$. In the following we only consider $\beta<\infty$. The case $\beta=\infty$ can be dealt with similarly. Choose $1<\theta<(\beta / \alpha)^{r /(1-r)}$. So $\beta-\alpha \theta^{(1 / r)-1}>0$. Note that $0<r<1$ as $r=d / 2$ and $0<d<2$. Now choose $\varepsilon>0$ such that

$$
\left((\beta-\varepsilon)-(\alpha+\varepsilon) \theta^{(1 / r)-1}\right)(\theta-1) \geqslant 2 \varepsilon .
$$

In particular, $\beta-\varepsilon \geqslant(\alpha+\varepsilon) \theta^{(1 / r)-1}$. By definition of $\beta$,

$$
\tau_{m} \geqslant(\beta-\varepsilon) m^{(1 / r)-1} \text { holds for infinitely many } m .
$$

We know from (a) and (b) that there exists $n_{0} \geqslant 1$ such that

$$
\tilde{T}_{j}^{m} \geqslant(\alpha-\varepsilon) T_{j}^{m} \text { for } m \geqslant n_{0} \text { and } 1 \leqslant j \leqslant m .
$$

Now choose $\tau_{n}$ satisfying (3.9) and $n-1 \geqslant n_{0}$. By Lemma 1.4 (i),

$$
\tau_{m} \geqslant \tau_{n} \geqslant(\beta-\varepsilon) n^{(1 / r)-1} \text { holds for } m \geqslant n .
$$

We claim that

$$
\widetilde{T}_{j}^{\lfloor\theta n\rfloor} \geqslant(\alpha+\varepsilon) T_{j}^{\lfloor\theta n\rfloor} \text { for all } 1 \leqslant j \leqslant\lfloor\theta n\rfloor .
$$

Once it is done, we have $\lfloor\theta n\rfloor \in S_{\alpha+\varepsilon}$. That is $S_{\alpha+\varepsilon} \neq \phi$. By (b), $\alpha \equiv \liminf _{n} \tau_{n} / n^{(1 / r)-1} \geqslant \alpha+\varepsilon$ and a contradiction follows.

Since $\beta-\varepsilon \geqslant(\alpha+\varepsilon) \theta^{(1 / r)-1},(3.11)$ implies that for $n \leqslant m \leqslant\lfloor\theta n\rfloor$,

$$
\tau_{m} \geqslant(\beta-\varepsilon) n^{(1 / r)-1} \geqslant(\alpha+\varepsilon)(\theta n)^{(1 / r)-1} \geqslant(\alpha+\varepsilon)([\theta n])^{(1 / r)-1} \geqslant(\alpha+\varepsilon) m^{(1 / r)-1} .
$$

Hence, (3.12) for $n \leqslant j \leqslant\lfloor\theta n\rfloor$ holds trivially. Moreover,

$$
\begin{aligned}
\tilde{T}_{n}^{\lfloor\theta n\rfloor}-(\alpha+\varepsilon) T_{n}^{\lfloor\theta n\rfloor} & \geqslant\left((\beta-\varepsilon) n^{(1 / r)-1}-(\alpha+\varepsilon)\lfloor\theta n\rfloor^{(1 / r)-1}\right)(\lfloor\theta n\rfloor-n+1) \\
& \geqslant\left((\beta-\varepsilon)-(\alpha+\varepsilon) \theta^{(1 / r)-1}\right)(\theta-1) n^{(1 / r)} \geqslant 2 \varepsilon n^{(1 / r)}
\end{aligned}
$$

by (3.8) and the inequality that $x-1 \leqslant\lfloor x\rfloor \leqslant x$. We have from (3.10) that for $j<n$,

$$
\tilde{T}_{j}^{n-1}-(\alpha+\varepsilon) T_{j}^{n-1} \geqslant-2 \varepsilon T_{j}^{n-1} \geqslant-2 \varepsilon T_{1}^{n-1} \geqslant-2 \varepsilon n^{(1 / r)-1} \cdot n=-2 \varepsilon n^{(1 / r)} .
$$

Adding up these two formulas above, we immediately obtain (3.12) for $1 \leqslant j<n$. This completes the proof of (3.12) and thus (c). 
$\operatorname{STEP}(\mathrm{d})$. By (c), $\lim _{n} \tau_{n} / n^{(1 / r)-1}=\alpha \in(0, \infty]$. Hence $\tau_{n} \approx \alpha n^{(1 / r)-1}$ for $n$ no less than some number $n_{0}$. Therefore,

$$
\begin{aligned}
\tilde{T}_{j}^{n} \approx \alpha \sum_{s=j}^{n} s^{(1 / r)-1} \approx \alpha \int_{j}^{n} s^{(1 / r)-1} d s & =\alpha r\left(n^{1 / r}-j^{1 / r}\right) \\
& =\alpha r\left(1-(j / n)^{1 / r}\right) n^{1 / r} \text { for } j \geqslant n_{0}
\end{aligned}
$$

Since $\max _{1 \leqslant j<n_{0}}\left(\tilde{T}_{j}^{n}\right)^{-r} \leqslant\left(\tilde{T}_{n_{0}}^{n}\right)^{-r} \leqslant\left(\left(n-n_{0}\right) \tau_{1}\right)^{-r} \stackrel{n}{\longrightarrow} 0$ by Lemma 1.4 (i), (1.2) implies

$$
1 \approx \sum_{j=n_{0}}^{n}\left(\tilde{T}_{j}^{n}\right)^{-r} \approx \sum_{j=n_{0}}^{n} \frac{1}{(\alpha r)^{r}\left(1-(j / n)^{1 / r}\right)^{r} n} \stackrel{n}{\longrightarrow} \frac{1}{(\alpha r)^{r}} \int_{0}^{1}\left(\frac{1}{1-x^{1 / r}}\right)^{r} d x .
$$

It implies $\alpha<\infty$ in particular. Letting $y=x^{1 / r}$ on the right above, we have ([3])

$$
\begin{aligned}
\lim _{n} \tau_{n} / n^{(1 / r)-1} & =\alpha=r^{-1+(1 / r)}\left(\int_{0}^{1}(1-y)^{-r} y^{r-1} d y\right)^{1 / r}=r^{-1+(1 / r)}(B(1-r, r))^{1 / r} \\
& =r^{-1+(1 / r)}(\Gamma(1-r) \Gamma(r) / \Gamma(1))^{1 / r}=r^{-1+(1 / r)}(\pi / \sin r \pi)^{1 / r}
\end{aligned}
$$

\section{REFERENCES}

[1] C.H. Chang, Y. Chow and Z. Wang, 'On the asymptotic behavior of heating times', Anal. Appl. (Singap.) 1 (2003), 429-432.

[2] Y.M. Chen, Y. Chow and J. Hsieh, 'On a heat conduction problem by Myshkis', J. Differ. Equations Appl. 6 (2000), 309-318.

[3] H. Hochstadt, The functions of mathematical physics (Wiley Interscience, New York, London, Sydney, 1971).

[4] A.D. Myshkis, 'On a recurrently defined sequence', J. Differ. Equations Appl. 3 (1997), 89-91.

Department of Mathematical Education

National Hualien Teachers College

Hualien

Taiwan 970

e-mail: jongyi@mail.nhltc.edu.tw
Institute of Mathematics

Academia Sinica

Taipei

Taiwan 115

e-mail: chow@math.sinica.edu.tw 